1 How distressing is referral to colposcopy in cervical cancer screening?

2 A prospective quality of life study

3

4 Ida J. Korfage ${ }^{1}$, PhD, assistant professor;

5 Marie-Louise Essink-Bot ${ }^{2}$, MD PhD, professor

6 Steven M. Westenberg ${ }^{3}, \mathrm{MD}$, gynecologist

7 Theo Helmerhorst ${ }^{4}, \mathrm{MD}$ PhD, professor, gynecologist

8 J. Dik F. Habbema ${ }^{1}$, PhD, professor

9 Marjolein van Ballegooijen ${ }^{1}, \mathrm{MD}$ PhD, associate professor

10

$11{ }^{1}$ Dept. of Public Health, Erasmus MC, University Medical Center Rotterdam, P.O. Box 2040,

123000 CA Rotterdam, the Netherlands

$13{ }^{2}$ Dept. of Public Health, Academic Medical Center, University of Amsterdam, P.O. Box 22660,

141100 DD Amsterdam, the Netherlands

$15{ }^{3}$ Dept. of Obstetrics and Gynaecology, Medical Center Alkmaar, P.O. Box 501, 1800 AM

16 Alkmaar, the Netherlands

$17{ }^{4}$ Dept. of Obstetrics and Gynaecology, Erasmus MC, University Medical Center Rotterdam,

18 P.O. Box 2040, 3000 CA Rotterdam, the Netherlands

19

20 Corresponding author: Ida J. Korfage

21 Erasmus MC, Dept. of Public Health

22 P.O. Box 2040, 3000 CA Rotterdam, the Netherlands

23 Tel. no.:+31 107038460

24 E-mail: i.korfage@erasmusmc.nl 
Objective: Referral for colposcopy because of abnormal Pap test results is likely to be distressing, but the extent and duration of these effects are unknown. We aimed to fill this gap.

Methods: We conducted a prospective observational study at two departments of Obstetrics and Gynecology (an academic and a non-academic setting). Women referred for colposcopy completed questionnaires before colposcopy, and at 1,3 , and 6 months afterwards. A reference group of 706 screen participants, aged 29-60 years old, was included and completed questionnaires once. Main outcome measures were generic health-related quality of life (HRQoL), assessed through the EQ-5D and the SF-12 physical and mental scores (PCS-12 and MCS-12); anxiety as assessed by STAI-6, and screen-specific anxiety as assessed by the Psychological Consequences Questionnaire (PCQ).

38 Results 154 women responded to the questionnaire, of whom 132 were included in the 39 analyses. Histological results were CIN 1 in 17/115 women (15\%) and CIN 2+ in 62 (54\%). In 36 40 women (31\%) there was no histologically confirmed neoplasia. Before colposcopy physical HRQoL scores were similar or slightly better than in the reference group, while mental HRQoL (MSC-12) and (screen-specific) anxiety were worse $(p<0.001)$. Irrespective of CIN-grades, anxiety washed out during follow-up ( $p<0.001)$, with changes being clinically relevant. Conclusions Referral for gynecological evaluation because of abnormal PAP-test results was distressing. Anxiety - and not the physical burden of management - seemed to be most bothersome to women. For all CIN-grades, distress disappeared over six months following colposcopy, suggesting a reassuring effect of gynecological management. 
$49 \quad$ Key words

50 - Cervical cancer;

$51 \quad$ - Longitudinal;

52 - Quality of life;

53 - Screening;

54 - Distress;

$55-$ CIN;

56 - cervical dysplasia;

$57 \quad-\quad$ abnormal Pap

58 
60 Screening for cervical cancer aims to reduce disease-specific mortality by early detection and treatment of pre-invasive (cervical intraepithelial neoplasia, CIN) or early invasive disease. Screen participants with abnormal Pap tests are generally referred for gynecological evaluation including colposcopy. Previous studies found that colposcopy was stressful for most women. (1) Not the procedure itself but the prospect of having cancer and risk of dying were the biggest sources of distress. (2)

66 Cervical cancer screening is aimed at preventing the disease by finding and treating precursor

67 lesions, but these precursors are known to often regress. (3) The number of treated precursors

68 will thus be considerably larger than the number of prevented cases of cervical cancer.

69 Screening policy thus requires balancing the benefits of preventing cancer by treatment of 70 lesions that are likely to resolve against the harms of screening. Distress and anxiety due to 71 screening are such harms. Until 2004 there had been little research on how short-term effects 72 of screening interventions affect quality of life. (4) While roughly half of the adult women in Europe are invited to have a smear test at least once every 5 years, of whom between 0.8 and $4.4 \%$ are referred to colposcopy every screening round, (5) the extent and duration of adverse quality of life effects after abnormal Pap test results are still unknown. We aimed to prospectively assess the effects of colposcopy referral on women's generic health-related quality of life (HRQoL) and on (screen-specific) anxiety levels. A female reference group of screen participants was included as a proxy of HRQoL levels preceding referral. We compared HRQoL and anxiety outcomes of the study group, referred to as

80 "colposcopy group", to those of the reference group. 


\section{Cervical cancer screening in the Netherlands}

84 In the Dutch national cervical cancer screening program, women aged 30-60 are invited once every 5 years to have a Pap test. Participation does not entail costs. At the time this study was conducted, the national uptake rate was 65\%, (6) and neither primary HPV screening nor HPV vaccination had been introduced. In 2009, $96.7 \%$ of women who participated had normal cytological smear results and in one percent Pap tests were of inadequate quality requiring repeat smears. High-grade cytological abnormalities, including moderately dyskaryotic (Pap $3 a 2$ (7)) or worse, were found in $0.5 \%$ to $0.7 \%$ and low grade abnormalities, including borderline or mildly dyskaryotic (Pap 2/3a1) smear results, were found in $1.8 \%$ of screen

92 participants. (6-8)

93 Women can be referred to gynecological evaluation through two different routes. Following

94 the screening protocol women whose smear results are moderately dyskaryotic (Pap 3a2) or 95 worse are immediately referred for colposcopy by a gynecologist. Women with borderline or mild dyskaryotic smear results (Pap2/3a1) are advised to have triage smears made by their GP.

97 (7) If these are once again abnormal women are also referred for colposcopy.

98 If histology results of biopsies taken at colposcopy indicate CIN-grade 2 or worse further treatment is performed. A more conservative approach is recommended for women diagnosed with CIN 1 since the majority of these lesions will regress. After two or three consecutive negative smears women with CIN 1 will return to the national screening program.

Study design

104 Between February 2006 and April 2008 a prospective longitudinal cohort study was conducted 105 in two Dutch hospitals. We aimed at including all women who were referred for gynecological 
106 evaluation because of abnormal Pap test results in the screening program. Women whose

107 patient files later showed that they were ineligible were excluded (see Figure 1.)

108 Women scheduled for colposcopy after abnormal smear results were sent a letter, in which

109 they were asked for written informed consent to participate in the study, which involved

110 completion of the attached questionnaire (see below), and 3 following ones after 1, 3 and 6

111 months (return envelopes were provided). Women were also asked for permission to consult

112 their patient files and/or the gynecologist for clinical data about colposcopy follow-up. They

113 were assured that not completing the questionnaires would not have any consequences for

114 their medical care. No reminders were sent after the initial questionnaire. Once women had

115 consented in participation in the study we sent reminders for follow-up questionnaires. A

116 group of screen participants was included as a reference (see below). Both groups were 29-60

117 years old.

118 This study was part of a comprehensive evaluation of the Dutch cervical cancer-screening

119 program. The medical ethics review committees of the Erasmus University Medical Center

120 Rotterdam (MEC-2004-099) and of Medical Center Alkmaar (M04-051) approved the research

121 protocol.

122

123 Respondents' characteristics

124 Questions on education, employment, marital status, and having children or not were part of

125 the initial questionnaire. Educational level was classified as low (primary school or lower

126 technical education), intermediate or high (college/university degree).

127 Information about Pap results at referral for gynecological evaluation and about CIN-grade was

128 available conditional on women having granted permission to consult their patient files and/or

129 gynecologist. 
130 In this paper all colposcopy results worse than CIN 1 will be referred to as CIN 2+. The most

131 severe grade of CIN in the first biopsy after inclusion in this study was used to define the

132 respondents' CIN-grades. (9)

133

134 Reference group

135 We compared HRQoL and anxiety scores of the intervention group to those of a reference

136 group of 706 screen participants, who had been recruited through the regional screening

137 organization in Maastricht (10). Data were collected after screening but before women knew

138 their test result. Reference and study group completed similar measures (see below).

139

140 Content of the questionnaires

141 Questionnaires included validated measures on generic HRQoL (11), generic anxiety (12), and

142 screen specific anxiety (13). Generic HRQoL was assessed through the EuroQol classification

143 (EQ-5D) and the 12-item Short-Form Health Survey (SF-12). The EQ-5D consists of 5 items

144 (mobility, self-care, usual activities, pain/ discomfort, and anxiety/ depression). Scores can be

145 linked to a utility score with 0 indicating 'death' and 1 'full health'. (14) The EQ-5D is

146 complemented by a visual analogue scale on current health, the Valuation of Own Health,

147 which is anchored by 'worst imaginable health state' $(0)$ and 'best imaginable health state'

148 (100). The SF-12 consists of 12 items in the physical and mental domain. Based on these item

149 scores summary measures for the physical and mental component (PCS-12 and MCS-12) are

150 constructed, (11) using norm-based methods with a mean of 50 and a standard deviation (SD)

151 of 10. Age- and sex-adjusted SF-12 norm scores from the Dutch population, including women

152 who do not participate in the screening program, are available from Statistics Netherlands.

$153 \quad(15)$ 
154 Generic anxiety was assessed through the STAI- 6 containing 6 items on e.g. feeling at ease or

155 upset. Higher scores (20-80) indicate higher levels of generic anxiety. $(12,16)$ STAI-State scores

156 of over 44 define an individual as highly anxious. (17)

157 Screen-specific anxiety was measured through the Psychological Consequences Questionnaire

158 (PCQ), which was developed to assess the consequences of breast screening on emotional,

159 physical, and social functioning. Corresponding subscales contain 5, 4, and 3 items,

160 respectively. (13) Ratings for symptoms within each dimension vary from 0 (not at all) to 3

161 (quite a lot of time). The overall PCQ score ranges from 0-36; (18) higher scores indicate more

162 dysfunction. We used the Dutch version as adapted by Rijnsburger and colleagues. (19)

163

164 Statistical analyses

165 In accordance with guidelines, (20) missing items in the STAI- 6 and the PCQ were imputed by

166 respondents' own average score if they had completed at least $50 \%$ of the items. Differences

167 between the colposcopy and reference groups considering background variables were

168 assessed using t-tests for continuous variables and Chi-square tests for categorical ones.

169 Differences considering HRQoL and anxiety scores were assessed using linear regression,

170 controlling for differences in age. A condition for linear regression is a normal distribution of

171 residuals. However, this condition is often not met when HRQoL measures are used. Therefore

172 we inspected the residuals and compared them with the normal distribution. The deviations

173 we found led us to perform a bootstrap analysis (21) (1,000 replicas) in the program $R,(22)$

174 while controlling for differences between groups in age.

175 Friedman tests were used to assess changes in HRQoL scores in the study group across

176 multiple measurements. Friedman tests are based on data from those who completed all

177 assessments. For each measure we report how many women completed it at all four time

178 points, and we report on the HRQoL and anxiety scores of just those women. We hypothesized 
179 that more anxiety would be reported at baseline if the initial Pap result was more serious.

180 Therefore we assessed HRQoL and anxiety by Pap result (Pap2/3a versus Pap 3b or worse),

181 using t-tests to assess the significance of the differences between groups. We also

182 hypothesized that the more serious the $\mathrm{CIN}$-grade turned out to be, the more anxiety and

183 screen specific anxiety would be reported at follow-up assessments, and therefore we

184 assessed HRQoL and anxiety per CIN group (i.e. no CIN was found versus CIN 1 versus CIN 2+).

185 We used ANOVA to assess the statistical significances of differences in HRQoL and anxiety

186 scores between CIN-groups. Statistical analyses were performed using SPSS for Windows,

187 version 17.

188 The minimal important difference (MID), indicating clinical relevance, was operationalized as a

189 difference of at least half a SD. (23)

190 
192154 women completed questionnaires after being referred for gynecological evaluation. Three

193 of them were too young to have participated in the screening program. We excluded them

194 from further analyses. After consulting patient files or gynecologists (if women had given us

195 permission to do so), we found that another 19 women were ineligible since they had not been

196 referred to the gynecologist after routine Pap tests $(n=15)$ or they had already been having

197 gynecological check-ups for at least a year $(n=4)$. Thus, 132 women were included for analysis

198 (see Figure 1, Table 1). Pap test results had been communicated to them by their GP (69\%), or

199 by their GP's assistant (29\%). In two cases the hospital informed these women. Women had

200 been contacted by telephone $(74 \%)$, in person $(22 \%)$ or by letter $(5 \%)$. There is no protocol

201 specifying how abnormal PAP results should be communicated to women.

202 Histological results were known in 115/132 women and were CIN 1 ( $n=17), \operatorname{CIN} 2$ ( $n=32$ ), CIN 3

203 ( $n=29)$, or carcinoma stage 1 ( $n=1)$. In 36 women there was no histologically confirmed

204 neoplasia. These women had been referred with Pap $2(n=21)$, Pap 3a $(n=13)$, or Pap 3b $(n=2)$.

205 In two women CIN-grades were unknown and fifteen women did not grant us permission to

206 access their patient files or gynecologist. Since their HRQoL and anxiety scores were similar to

207 those who had routine cervical smears, we included them in the analyses. Management was

208 known in 117 women. Forty-six out of these women did not receive therapy, , 60/117 were

209 treated once and 11 women were treated more than once (11/117), e.g. by LLETX excision

210 and conisation or they had conisation twice. Table 2 presents the most invasive therapy per

211 woman, reported per CIN-grade.

212 Overall, questionnaire response rates were $114(86 \%), 110(83 \%)$, and $108(82 \%)$ at 1, 3, and 6

213 months follow-up.

215 Comparison colposcopy group and reference group 
216 Background variables differed significantly between the colposcopy and the reference group

217 (Table 1). As expected, women referred for colposcopy $(n=132)$ were younger (40.6 vs. 45.6

218 years), because low grade CIN is more prevalent in younger age groups. Compared to the

219 reference group they had more often paid jobs and less often children.

220 The crude PCS-12 scores of the colposcopy group were significantly higher - which indicates

221 better physical functioning - than those of the reference group (54 versus 51 , Table 3 ) and than

222 the age adjusted norm score of 51 for the female Dutch population (Statistics Netherlands).

223 The MCS-12 scores of the colposcopy group, however, were lower - which indicates poorer

224 mental functioning - than those of the reference group (47 versus 53, Table 3 ) and than the

225 Dutch norm scores of 52 (Statistics Netherlands). Differences remained significant after

226 controlling for age (Table 3).

227 Average crude STAI-6 and PCQ scores were higher in the colposcopy group than in the

228 reference population, indicating more generic and screen specific anxiety in women with

229 abnormal smear results. Differences in STAI-scores and in two PCQ subscale scores exceeded

230 the Minimal Important Difference (MID), indicating that the differences between the

231 colposcopy group and the reference population were of clinical relevance (Table 3).

232 For all scale scores bootstrap analyses resulted in similar conclusions considering statistical

233 significance and clinical relevance as the linear regression analyses.

234

235

Generic HRQoL and anxiety: results over time

236 Changes over time in the EQ-5D utility score, the EQ-5D 'rating of own health', and the sum

237 score for physical function (PCS-12) were neither statistically significant nor clinically relevant.

238 The scores for mental health score (MSC-12), generic anxiety (STAI-6), and screen-specific

239 anxiety (PCQ) improved over time ( $p<0.001)$. Overall, changes over time indicated improved

240 functioning towards the end of the follow up period. Changes in generic anxiety and in two 
241 subscales of screen-specific anxiety indicated clinical relevance (Table 4). At baseline, $32 \%$ of

242 the colposcopy group (41/130) reported high anxiety levels (i.e. STAI-6 scores of over 44). This

243 decreased to $18 \%(20 / 112)$ at 1 month follow-up, and to $14 \%$ at 3 and 6 months follow-up

$244(15 / 110$ and $15 / 108$, respectively). High anxiety was reported by $10 \%$ of the reference group.

245 The significance of the difference between the groups decreased from $p<0.001$ at baseline to

$246 \quad 0.24$ at 6 months follow-up.

247 At 6 months follow-up, HRQoL and generic anxiety scores of the colposcopy group were similar

248 to those of the reference group, while screen-specific anxiety scores remained worse.

249

250 Generic HRQoL and anxiety over time by initial Pap test result

251 HRQoL and anxiety were similar in women referred for colposcopy with Pap 2/Pap 3a (at most

252 moderately dysplastic, $n=90$ ) versus women with Pap 3b or worse (at least severely dysplastic, $253 \mathrm{n}=21)$, data not shown.

254

255 Generic HRQoL and anxiety over time by CIN-grade

256 In 115 cases CIN-grades were known. Regardless of CIN-grade, generic HRQoL remained at

257 similar levels throughout follow-up and (screen specific) anxiety decreased over time (Figure

258 2). With two exceptions, HRQoL and anxiety scores differed significantly between the $3 \mathrm{CIN}$ -

259 groups.

260 


\section{Discussion}

262 We assessed the HRQoL and anxiety in a cohort of women with abnormal Pap test results who

263 were referred for gynecological evaluation. At baseline, the colposcopy group reported more anxiety than the reference group, with differences being clinically relevant. We found that during follow-up, overall, HRQoL improved in the colposcopy group and their anxiety decreased over time, irrespective of CIN-grade.

267 The availability of clinical data, which enabled us to discriminate between varying degrees of abnormalities, is one of the strengths of this prospective study. Also, as recommended for quality of life research, we used both generic and screen-specific health measures that had been validated in similar groups as the currently described population. To enable interpretation of the HRQoL and anxiety scores we included a reference group, which is recommended but not often done (4). Limitations of this study are the lack of data about the length of the interval between the receipt of the Pap test results and the colposcopy results, the response rate being unavailable, and the relatively low number of respondents who were diagnosed with CIN 1.

CIN grade 2+ was found in 62 out of 115 women and the positive predictive value (PPV) was thus $54 \%$, which is comparable to the $49 \%$ PPV of a moderately dyskaryotic Pap test in the Dutch screening program (5). In 36 women in our cohort (31\%) only normal Pap tests and histology results were observed during follow-up. These so-called false positive test results are inherent to screening programs;

283 an abnormal test result leads to additional tests and hospital visits and may cause anxiety or 284 worry, while no abnormalities are found in the end. This group of women, of whom four 285 received treatment, reported similar HRQoL and higher anxiety levels as who were found to 
have $\mathrm{CIN} 2+$, while in the latter group 59 out of 62 women received treatment. Anxiety - and not the physical burden of management - seemed to be most bothersome to women. In a review of 210 papers Cullen et al. concluded that affected domains in women with falsepositive screening results include distress, fear and worry about having or getting cancer. (4) This issue becomes even more relevant with the introduction of HPV-screening, since the specificity of HPV screening is expected to be considerably lower in younger age groups (24). Twenty years ago most women interpreted the term precancer as 'early cancer'. (25) Also more recently, mildly abnormal smear results were misinterpreted as actually having cancer

$294(26,27)$ which will lead to more anxiety. $(27)$ We therefore recommend to provide women who have abnormal smear test results with clear written information about the meaning of this result, stressing that the abnormal test result does not indicate that they have cancer, and

297 to check in person or by phone whether this information was properly understood. In a previous study, women not complying with follow-up protocols reported the

299 highest anxiety scores. (28) Since we only included women who did participate in follow-up 300 protocols, we probably arrived at an underestimation of women's anxiety, even more so because pathologically high levels of anxiety and worry apparently lead to low screening rates.

304 of a lasting or serious nature in the majority of women. $(29,30)$ However, in a cross-sectional

305 study among 270 women, addressed at 6-24 months after the initially abnormal Pap test

306 result, our research group showed that borderline and mildly dyskaryotic smear results were

307 consistently associated with considerable excess anxiety. (31) 
310 We conclude that referral for gynecological evaluation after abnormal PAP-test results

311 negatively impacted mental health. Anxiety - and not the physical burden of management -

312 seemed to be most bothersome to women, which confirms earlier literature. Irrespective of

313 CIN-grade, this negative effect on mental health diminished over time and had washed out at 6

314 months after baseline. Possibly, this indicates that management had a reassuring effect and

315 led to reduced anxiety levels. We recommend carefully choosing cut-off strategies for referral

316 to colposcopic evaluation. Also, clear communication about the meaning of false-positive test

317 results is needed with women invited to participate in screening and with women who have

318 abnormal test results, so they will understand what is going on - and especially what is not. 


\section{Acknowledgments}

320 We are grateful to the women who completed questionnaires for participating in the study.

321 We thank the staff at the Departments of Obstetrics and Gynecology in Medical Center

322 Alkmaar and in Erasmus MC for their friendly cooperation.

323

324

\section{Disclosure of interest}

Until 2008, Dr. M. van Ballegooijen was principal investigator for a project on the costeffectiveness of human papilloma virus vaccination, financed through an unrestricted grant by GSK (a pharmaceutical company that produces human papilloma virus vaccines against cervical cancer). The authors received funding from charities and from governmental and public bodies, including the National Institute for Public Health and the Environment, to conduct their research.

\section{Contribution to authorship}

M. van Ballegooijen, D. Habbema and M.L. Essink-Bot conceived the idea for the study; M. van Ballegooijen and M.L. Essink-Bot designed the protocol; M.L. Essink-Bot supervised the execution of the study; I. Korfage, M. van Ballegooijen and M.L. Essink-Bot designed the questionnaire; I. Korfage, S. Westenberg and T. Helmerhorst organized the local data collection; I. Korfage was responsible for the database design and data entry; I. Korfage, M. van Ballegooijen, and M.L. Essink-Bot made the statistical design; I. Korfage performed the analyses; I. Korfage drafted the report; all the collaborators listed above contributed/edited the paper. I. Korfage is the guarantor of the study.

341 All authors have full access to all of the data in the study and can take responsibility for the 342 integrity of the data and the accuracy of the data analysis. 


\section{Details of ethics approval}

346 The medical ethics review committees of the Erasmus University Medical Center Rotterdam

347 (MEC-2004-099) and of Medical Center Alkmaar (M04-051) approved the research protocol.

348 All participating women gave written informed consent.

349

\section{$350 \quad$ Funding}

351 We gratefully acknowledge the Dutch Cancer Society for funding our research to evaluate the

352 Dutch national cervical cancer screening program, including Quality of Life effects (EMCR

353 2003-2775). The authors were completely independent from funders in conducting and

354 writing this study.

355

Legends of tables and figures

356

Figure 1 Flowchart of study population

357

Table 1 Background characteristics of the colposcopy group, observed scores in numbers and percentages, unless otherwise indicated, compared with a reference group of screen

359 participants.

360

Table 2 Most invasive treatment per woman, reported per CIN-grade

361

Table 3 Generic Quality of Life scale scores (SD) in women referred to the gynecologist for colposcopy, shortly after their abnormal test results and in a reference population of screen participants. Statistical significance of differences between groups was ageadjusted.

Table 4 Time trend analysis (repeated measures) of women with an abnormal Pap test result (colposcopy group); starting before the first consultation with the gynecologist, plus 

changes over that time period.

369 Figure 2 Health-related quality of life and anxiety scores per CIN-stage at four assessments.

370

371 
373 1. Tahseen S, Reid PC. Psychological distress associated with colposcopy: patients' 374 perception. Eur J Obstet Gynecol Reprod Biol. 2008;139:90-4.

375 2. Valdini A, Esielionis P. Measurement of colposcopy-associated distress using the 376 psychosocial effects of having an abnormal pap smear-questionnaire in a Latina population. J 377 Low Genit Tract Dis. 2004;8:25-32.

378 3. Holowaty P, Miller AB, Rohan T, To T. Natural history of dysplasia of the uterine cervix. 379 Journal of the National Cancer Institute. 1999;91:252-8.

380 4. Cullen J, Schwartz MD, Lawrence WF, Selby JV, Mandelblatt JS. Short-term impact of 381 cancer prevention and screening activities on quality of life. J Clin Oncol. 2004;22:943-52.

382 5. Ronco G, van Ballegooijen M, Becker N, Chil A, Fender M, Giubilato P, et al. Process 383 performance of cervical screening programmes in Europe. Eur J Cancer. 2009;45:2659-70.

384 6. Erasmus MC. Screening program cervical cancer, report on 2009 [bevolkingsonderzoek 385 baarmoederhalskanker, LEBA rapportage 2009]2011: Available from:

386 http://www.rivm.nl/bevolkingsonderzoeknaarkanker/images/2slagfolder final.pdf.

387 7. Rebolj M, van Ballegooijen M, Berkers LM, Habbema D. Monitoring a national cancer 388 prevention program: successful changes in cervical cancer screening in the Netherlands. Int J 389 Cancer. 2007;120:806-12.

390 8. Berkers LM, van Ballegooijen M, van Kemenade FJ, Rebolj M, Essink-Bot ML, 391 Helmerhorst TJ, et al. [The 1996 revision of the Dutch cervical cancer screening programme:

392 increased coverage, fewer repeat smears and less opportunistic screening]

393 Herziening bevolkingsonderzoek op baarmoederhalskanker 1996: hogere dekkingsgraad,

394 minder herhalingsuitstrijkjes en minder opportunistische screening. Ned Tijdschr Geneeskd.

395 2007;151:1288-94.

396 9. Insinga RP, Glass AG, Myers ER, Rush BB. Abnormal outcomes following cervical cancer 397 screening: event duration and health utility loss. Med Decis Making. 2007;27:414-22.

398 10. Korfage I, van Ballegooijen M, Wauben B, Looman C, Habbema J, Essink-Bot ML.

399 Having a Pap smear, quality of life before and after cervical screening: a questionnaire study.

400 BJOG. 2012;119:936-44.

401 11. Gandek B, Ware JE, Aaronson NK, Apolone G, Bjorner JB, Brazier JE, et al. Cross-

402 validation of item selection and scoring for the SF-12 Health Survey in nine countries: results

403 from the IQOLA Project. International Quality of Life Assessment. J Clin Epidemiol.

404 1998;51:1171-8. 

dutch short form of the state scale of the Spielberger State-Trait Anxiety Inventory: considerations for usage in screening outcomes. Community Genet. 2003;6:84-7. 13. Cockburn J, De Luise T, Hurley S, Clover K. Development and validation of the PCQ: a questionnaire to measure the psychological consequences of screening mammography. Soc Sci Med. 1992;34:1129-34.

411 14. Dolan P. Modeling valuations for EuroQol health states. Med Care. 1997;35:1095-108.

412 15. Central Bureau of Statistics. Reported health and lifestyle (in Dutch). Centraal Bureau

413 voor de Statistiek. Voorburg/Heerlen, the Netherlands2008 [updated August 6th, 2008];

414 Available from: $\underline{\text { http://statline.cbs.nl/. }}$

415 16. Marteau TM, Bekker H. The development of a six-item short-form of the state scale of 416 the Spielberger State-Trait Anxiety Inventory (STAI). Br J Clin Psychol. 1992;31:301-6.

417 17. Millar K, Jelicic M, Bonke B, Ashbury AJ. Assessment of preoperative anxiety:

418 comparison of measures in patients awaiting surgery for breast cancer. Br J Anaesth. 419 1995;74:180-3.

420 18. Ong G, Austoker J, Brett J. Breast screening: adverse psychological consequences one 421 month after placing women on early recall because of a diagnostic uncertainty. A multicentre 422 study. J Med Screen. 1997;4:158-68.

423 19. Rijnsburger AJ, Essink-Bot ML, van As E, Cockburn J, de Koning HJ. Measuring

424 psychological consequences of screening: adaptation of the psychological consequences 425 questionnaire into Dutch. Qual Life Res. 2006;15:933-40.

426 20. Ware JEJ, Snow KK, Kosinski M, Gandek BG. SF-36 health survey: manual and 427 interpretation guide: Boston, MA : The Health Institute, New England Medical Center; 1993.

428 21. Efron B, Tibshirani R. An Introduction to the Bootstrap. New York Chapman \& Hall; 4291993.

430 22. R Development Core Team. R: A language and environment for statistical computing. R 431 Foundation for statistical computing, editor. Vienna, Austria 2008.

432 23. Norman GR, Sloan JA, Wyrwich KW. Interpretation of changes in health-related quality 433 of life: the remarkable universality of half a standard deviation. Med Care. 2003;41:582-92.

434 24. Wright TC, Jr., Schiffman M, Solomon D, Cox JT, Garcia F, Goldie S, et al. Interim 435 guidance for the use of human papillomavirus DNA testing as an adjunct to cervical cytology 436 for screening. Obstet Gynecol. 2004;103:304-9. 
437 25. Wilkinson C, Jones JM, McBride J. Anxiety caused by abnormal result of cervical smear 438 test: a controlled trial. BMJ (Clinical research ed. 1990;300:440.

439 26. Hounsgaard L, Petersen LK, Pedersen BD. Facing possible illness detected through

440 screening--experiences of healthy women with pathological cervical smears. Eur J Oncol Nurs.

$441 \quad 2007 ; 11: 417-23$.

442 27. Jones $\mathrm{MH}$, Singer A, Jenkins $\mathrm{D}$. The mildly abnormal cervical smear: patient anxiety and 443 choice of management. J R Soc Med. 1996;89:257-60.

444 28. Lerman C, Miller SM, Scarborough R, Hanjani P, Nolte S, Smith D. Adverse psychologic 445 consequences of positive cytologic cervical screening. Am J Obstet Gynecol. 1991;165:658-62.

446 29. Drolet M, Brisson M, Maunsell E, Franco EL, Coutlee F, Ferenczy A, et al. The 447 psychosocial impact of an abnormal cervical smear result. Psychooncology. 2012;21:1071-81.

448 30. Reelick NF, de Haes WF, Schuurman JH. Psychological side-effects of the mass

449 screening on cervical cancer. Soc Sci Med. 1984;18:1089-93.

450 31. Korfage IJ, van Ballegooijen M, Huveneers H, Essink-Bot ML. Anxiety and borderline 451 PAP smear results. Eur J Cancer. 2010;46:134-41.

452

453

454 
Table 1 Background characteristics of the colposcopy group, observed scores in numbers and

456 percentages, unless otherwise indicated, compared with a reference group of screen participants.

\begin{tabular}{|c|c|c|c|}
\hline & $\begin{array}{l}\text { Colposcopy group } \\
\qquad n=132\end{array}$ & $\begin{array}{c}\text { Screen } \\
\text { participants } \\
n=706\end{array}$ & $p$-value \\
\hline Age (years) & & & $<0.001$ \\
\hline Average (SD) & $40.6(8.2)$ & $45.6(9.3)$ & \\
\hline Median & 40.2 & 45.1 & \\
\hline Range & $29-60$ & $29-60$ & \\
\hline Missing & 4 & 1 & \\
\hline Education (\%) & & & 0.06 \\
\hline Low education & $21(17)$ & $144(23)$ & \\
\hline Medium & $77(62)$ & $323(50)$ & \\
\hline High & $26(21)$ & $174(27)$ & \\
\hline Missing & 8 & 65 & \\
\hline Employment status (\%) & & & 0.03 \\
\hline Paid job & $92(81)$ & $419(67)$ & \\
\hline Housewife/unpaid job/student & $16(14)$ & $142(23)$ & \\
\hline No job & $6(5)$ & $49(8)$ & \\
\hline Retired & 0 & $13(2)$ & \\
\hline Missing & 18 & 83 & \\
\hline Marital status (\%) & & & 0.03 \\
\hline Married/cohabiting & $92(72)$ & $567(81)$ & \\
\hline Living without partner & $36(28)$ & $137(20)$ & \\
\hline Missing & 4 & 2 & \\
\hline Children (\%) & & & 0.002 \\
\hline No & $40(32)$ & $130(20)$ & \\
\hline Yes & $84(68)$ & $528(80)$ & \\
\hline Missing & 8 & 48 & \\
\hline Average no. of children & 2 & 2 & \\
\hline Country of birth (\%) & & & $<0.001$ \\
\hline the Netherlands & $120(92)$ & 627 (99) & \\
\hline otherwise & $11(8)$ & $4(1)$ & \\
\hline Missing & 1 & 64 & \\
\hline
\end{tabular}

457 
Table 2 Most invasive treatment per woman, reported per CIN-grade

460

\begin{tabular}{|c|c|c|c|c|c|c|}
\hline & & Most invasive $t$ & per woman, & & No & \\
\hline & Cryotherapy & LLETZ excision & Conisation & Uterus extirpation & therapy & Total \\
\hline CIN-grade & & & & & & \\
\hline No neoplasia found & $1(1 \%)$ & $0(-)$ & $1(1 \%)$ & $2(2 \%)$ & $32(27 \%)$ & $36(31 \%)$ \\
\hline $\mathrm{CIN}=1$ & $4(3 \%)$ & $2(2 \%)$ & $1(1 \%)$ & $0(-)$ & $10(9 \%)$ & $17(15 \%)$ \\
\hline CIN2+ & $20(17 \%)$ & $29(25 \%)$ & $7(6 \%)$ & $3(3 \%)$ & $3(3 \%)$ & $62(53 \%)$ \\
\hline Unknown CIN-grade & $0(-)$ & $1(1 \%)$ & $0(-)$ & $0(-)$ & $1(1 \%)$ & $2(2 \%)$ \\
\hline Total & 25 (21\%) & 32 (27\%) & $9(8 \%)$ & $5(4 \%)$ & 46 (39\%) & $117(100 \%)$ \\
\hline
\end{tabular}

461

462

463 
Table 3 Generic Quality of Life scale scores (SD) in women referred to the gynaecologist for colposcopy, shortly after their abnormal test results and in a reference population of screen participants. Statistical significance of differences between groups was age-adjusted.

\begin{tabular}{|c|c|c|c|}
\hline & $\begin{array}{l}\text { Colposcopy } \\
\text { group } \\
n=132\end{array}$ & $\begin{array}{c}\text { Screen } \\
\text { participants } \\
n=706\end{array}$ & $p$-value \\
\hline \multicolumn{4}{|l|}{ Generic health-related quality of life } \\
\hline EuroQol utility, EQ-5D (0-1) & $0.90(0.14)$ & $0.90(0.18)$ & 0.85 \\
\hline EuroQol , Rating of own health $(0-100)$ & $80(12)$ & $81(13)$ & 0.46 \\
\hline \multicolumn{4}{|l|}{ SF-12 (0-100) } \\
\hline Sumscore physical (PCS-12) & $54(8)$ & $51(10)$ & 0.04 \\
\hline Sumscore mental (MCS-12) & $47(12)$ & $53(9)$ & $<0.001$ \\
\hline \multicolumn{4}{|l|}{ Generic Anxiety } \\
\hline STAI-6 $(20-80) *$ & $41(12)$ & $33(10)$ & $<0.001$ \\
\hline Range & $20-73$ & $20-77$ & \\
\hline Highly anxious (STAl score >44), n (\%) & $41(32 \%)$ & $70(10 \%)$ & $<0.001$ \\
\hline \multicolumn{4}{|l|}{ Screen-Specific Anxiety } \\
\hline \multicolumn{4}{|l|}{ PCQ } \\
\hline Emotional Scale (0-15) & $4(4)$ & $1(2)$ & $<0.001$ \\
\hline Physical Scale (0-12) * & $2(2)$ & $0(1)$ & $<0.001$ \\
\hline Social Scale (0-9) * & $2(2)$ & $0(1)$ & $<0.001$ \\
\hline Total Score (0-36) * & $8(7)$ & $2(4)$ & $<0.001$ \\
\hline Range Total Score & $0-29$ & $0-30$ & \\
\hline
\end{tabular}

469 EuroQol and SF-12: higher scores indicate better functioning

470 STAI-6 and PCQ: higher scores indicate worse functioning.

$471 *$ differences exceeded the minimal important difference (MID), indicating clinical relevance 
473 Table 4 Time trend analysis (repeated measures) of women with an abnormal Pap test result

474 (colposcopy group); starting before the first consultation with the gynaecologist, plus follow-up

475 assessments at 1, 3, and 6 months later, and the statistical significance of changes over that

476 time period.

\begin{tabular}{|c|c|c|c|c|c|c|}
\hline $\begin{array}{l}\text { Shortly after } \\
\text { suspicious } \\
\text { smear } \\
n=132\end{array}$ & $\begin{array}{l}\text { At } 1 \text { month } \\
\text { follow-up } \\
n=114\end{array}$ & $\begin{array}{c}\text { At } 3 \text { months } \\
\text { follow-up } \\
n=110\end{array}$ & $\begin{array}{c}\text { At } 6 \text { months } \\
\text { follow-up } \\
n=108\end{array}$ & $\begin{array}{c}p \text { - } \\
\text { value* }\end{array}$ & $\begin{array}{c}\text { No. of } \\
\text { women who } \\
\text { completed } \\
\text { measure all } \\
4 \text { times }\end{array}$ & $\begin{array}{c}\text { Inter- } \\
\text { pretation }\end{array}$ \\
\hline $0.91(0.14)$ & $0.90(0.15)$ & $0.93(0.15)$ & $0.90(0.21)$ & 0.16 & 95 & Similar \\
\hline $81(12)$ & $77(18)$ & $80(17)$ & 78 (18) & 0.08 & 95 & Similar \\
\hline
\end{tabular}

SF-12 (0-100)

Sumscore physical (PCS-12)

Sumscore mental (MCS-12)

$\begin{array}{ccccccc}54(9) & 53(9) & 53(8) & 53(10) & 0.27 & 77 & \text { Similar } \\ 50(10) & 49(11) & 52(10) & 53(9) & <0.001 & 77 & \text { Improved }\end{array}$

Generic Anxiety

STAI-6 (20-80) *

$40(11) \quad 37(13) \quad 33(9)$

$34(10) \quad<0.001$

96

Improved

Screen-specific Anxiety

PCQ

Emotional Scale (0-15)

Physical Scale (0-12) **

\begin{tabular}{llllllll}
$5)$ & $4(4)$ & $3(3)$ & $2(3)$ & $2(3)$ & $<0.001$ & 96 & Improved \\
$* *$ & $2(2)$ & $2(3)$ & $1(2)$ & $1(2)$ & $<0.001$ & 96 & Improved \\
& $2(2)$ & $2(2)$ & $1(2)$ & $1(2)$ & $<0.001$ & 96 & Improved \\
$7(7)$ & $6(7)$ & $4(6)$ & $3(6)$ & $<0.001$ & 96 & Improved \\
\hline
\end{tabular}

Social Scale (0-9) *

Total Score (0-36) *

$477 *$ Statistical significance of differences was calculated using Friedman tests, including only respondents

478 that completed all four assessments. HRQoL and anxiety scale scores are reported of those who

479 completed that specific scale at each assessment.

$480 * *$ Differences between first and fourth assessment exceed the minimal important difference (MID),

481 indicating clinical relevance

482

483 EuroQol and SF-12: higher scores indicate improved functioning

484 STAI-6 and PCQ: higher scores indicate poorer functioning.

485 
Figure 1. Flowchart of study population

Women who were referred to a gynecologist because of abnormal Pap smear results were addressed with a baseline questionnaire.

154 consecutive patients completed the baseline questionnaire.

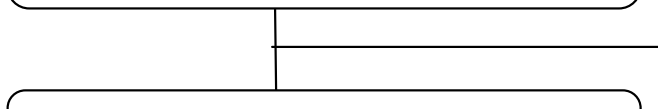

132 women were included in the analyses, of whom 117 granted us permission to access their files and/or their treating gynecologist.

114 of the 132 women (86\%) completed the second assessment at 1 month after baseline.

110 of the 132 women $(83 \%)$ completed the third assessment at 3 months after baseline.

108 of the 132 women (82\%) completed the fourth assessment at 6 months after baseline.
22 women were excluded from analyses since they had not been referred for gynecological evaluation because of a recent abnormal Pap test result $(n=19)$, or their age was below the threshold of the national cervical cancer screening program $(n=3)$. 